\title{
Effect of Ordering Policies on Complex Dynamics Behaviours in a Discrete-Type Manufacturing Industry Supply Chain System
}

Wen Wang ${ }^{*}$ and Wei-Ping Fu

\begin{abstract}
The beer game model is a typical paradigm used to study complex dynamics behaviours in production-distribution systems. The model, however, does not accord with current practical supply chain system models in discrete-type manufacturing industry, which are generally composed of retailers, distributors, manufacturers with internal supply chain, and suppliers. To describe how ordering policies influence the complex dynamics behaviour modes and operating cost in a general discrete-type manufacturing industry supply chain system, a high dimension piecewiselinear dynamics model is built for the supply chain system. Five kinds of ordering policy combination are considered. The distribution of both the largest Lyapunov exponent of effective inventory and average operating cost per cycle is obtained by simulation in a policy space. The simulation shows that for the general discrete-type manufacturing industry supply chain system, the upper chaotic corners emerge besides the lower chaotic corners in the policy space expressing the distribution of system behaviour mode, and that the ordering policies at each supply chain node as well as their combination have very significant effect on the topology of the distribution of both system behaviour mode and operating cost in the policy space. We find that chaos is not always corresponding to high cost, and the "chaos amplification" is not completely relevant to the "cost amplification".
\end{abstract}

Keywords: Supply chain system, Ordering policy, Operating cost, Nonlinear dynamics behavior

\section{Introduction}

The beer game model is the earliest, the most simple and complete model used to study complex nonlinear dynamics behaviours in supply chains. The beer game was derived from the product distribution game developed by Forrester [1] in the process of studying factory dynamics, which simulates the decision-making process of ordering or stocking in a chain-type production-distribution system consisting of such 4 sectors (nodes) as a retailer, a wholesaler, a distributor and a factory in total. The beer game model was early taken only as a universal case in investigating the bullwhip effect in supply chains, however, it has been considered as a classical model with nonlinear dynamics in supply chain systems since Mosekilde et al. [2] and Sterman [3] found chaos phenomenon in

\footnotetext{
*Correspondence: wangwen@xaut.edu.cn

Faculty of Mechanical and Precision Instrument Engineering, Xi'an University of Technology, Xi'an 710048, China
}

the game. The previous researches [2-11] have shown that complex nonlinear dynamics behaviours, e.g., chaos/ hyperchaos, can occur in the production-distribution system represented by the beer game model. The nonlinear factors come mainly from the nonlinear coupling of several oscillating feedback produced by the feedback structures among different decision-making entities, multiple inventory levels, time lag, etc., as well as the non-negativity $[10,12,13]$ and piecewise-linearity [14] in ordering/dispatching decision-making. The chaos complex dynamics behaviours have been observed in multilevel series supply chain inventory systems [15-17], production-recycling system [18] and customer-supplier systems [19-22] more simple than the beer game model.

There are many factors influencing complex dynamics behaviours of supply chain systems $[7,10,11,19,20$, 23-28], including operating policies for ordering, production and sales, etc., as well as demand pattern, lead time, demand-information sharing, supply chain levels, 
etc. of which ordering policy is the most basic affecting factor. The most important effect of nonlinearity on the ordering or inventory policy of production-distribution systems is that the chaos solutions exist in significant policy parameter space to management, i.e., the chaotic corner $[4,7]$. The more practical significance is that there are direct relations between the nonlinear dynamics behaviours of the system and the operating cost $[7,16]$. Inside the chaotic corner area, the chaotic and hyperchaotic behaviours might intuitively be expected to generate the highest operating cost as these types of behaviour are associated with large oscillations [7]. In addition, the complex behaviour mode distribution in policy parameter space of the supply chain system is different as the managers use various ordering policies, for example, the fixed expected inventory $[5,6,8,12]$ and the variable expected inventory $[7,10]$.

In the above studies, the original beer game model $[2,3]$ was usually adopted, and the single heuristic ordering policy [12] was basically used by each node in most models of them. In this paper, based on the original beer game model, a more general supply chain system model in discrete-type manufacturing industry is considered, which consists of retailers, distributors, manufacturers and suppliers. Both the largest Lyapunov exponent of effective inventory and average operating cost at each node are obtained by simulation. The effect of different ordering policy combination is studied on the distribution of the dynamics behaviour mode (expressed by the largest Lyapunov exponent) and the corresponding average operating cost.

The rest parts of this paper are organized as follows: Section 2 describes the structure model and the operating mechanism of the supply chain system, and builds the dynamics model of the system; Section 3 describes the ordering policy and operating cost at each node; Section 4 simulates the supply chain system benchmark model; Section 5 analyses the effect of different ordering policies and their combination on the distribution of the dynamics behaviour mode and the corresponding average operating cost; Section 6 concludes.

\section{Supply Chain System Model}

\subsection{Structure Model and Operating Mechanism of the Supply Chain System}

Being different from the original beer game model, the supply chain system in this paper consists of 4 nodes, i.e., retailer, distributor, manufacturer and supplier, as shown in Figure 1, of which there exist sectors for purchasing, production and marketing in the manufacturer.

For the convenience of analysis, it is assumed that the supply chain only supplies one type of product, and customer demand is exogenous $[7,10]$. Each node of the supply chain is governed by the following rules in each

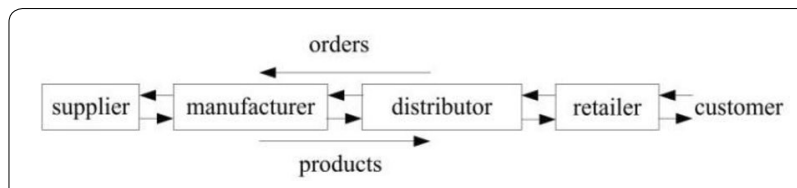

Figure 1 Simplified structural model of supply chain in discrete-type manufacturing industry

period (to be referred to as a week): (1) receiving orders from downstream node, filling orders as possible according to current inventory and shipping goods, last round stock out being filled at this round; (2) checking inventory, determining next round order quantity and sending purchase orders to upstream node, and placed orders not being cancelled; (3) receiving goods from upstream node, or producing and replenishing stocks, and shipments made not being returned.

In terms of the above rules, each node of the supply chain uses the same mechanism for orders' receiving and sending, as well as goods' receiving and shipping. In addition, the manufacturer will make a production decision (i.e., production orders of product) based on the distributor's order, demand prediction and inventory information, organize production and purchase raw material and parts for products from the supplier. These tasks will be respectively finished by every sector in the inner supply chain of the manufacturer, i.e., sales department, production department and purchasing department. The supplier will determine production of the raw material and parts based on the manufacturer's orders and other relative information, and carry out production and supply.

In order to compare with the simulation of the original beer game model, in despite of differences from the structure of most beer game models, we suppose in the model of this paper that the production and shipment of goods and the transmission of orders involve time delays; and the production capacity of the manufacturer and the supplier is unlimited. The production time is two time periods, and both the transmission delay of orders and the shipment delay of the product between two successive levels of the outer supply chain are one time period, while there is no time delay in the transmission of orders and goods between two successive sectors of the inner supply chain of the manufacturer.

\subsection{Heuristic Ordering Policy}

In the process implementing orders, the only decision variable for all supply chain nodes is the order quantity to the upstream node in each period. The basic ordering behaviour policy used in this study is a heuristic order mechanism $[7,10,12]$. To facilitate the mechanism description, the following notations are introduced in Table 1. 


\section{Table 1 Notations}

\begin{tabular}{|c|c|}
\hline Symbol & Description \\
\hline$t$ & Time or period \\
\hline$\hat{D}_{t}$ & The expected demand \\
\hline$D_{t}$ & The actual demand i.e. the incoming orders \\
\hline It & The actual inventory level \\
\hline$l_{t}^{*}$ & The desired inventory level \\
\hline$A l_{t}$ & The adjustment for the inventory level \\
\hline$S L_{t}$ & The actual supply line (orders placed but not yet received) \\
\hline$S L_{t}^{*}$ & The desired supply line \\
\hline$A S L_{t}$ & Adjustment for the supply line \\
\hline$O_{t}$ & Order quantity \\
\hline$\delta$ & The desired coverage \\
\hline$\gamma$ & $\begin{array}{l}\text { The expected (or perceived) lag between ordering and acqui- } \\
\text { sition of goods }\end{array}$ \\
\hline$\theta$ & $\begin{array}{l}\text { A constant which determines how fast expectations are } \\
\text { updated, } 0 \leq \theta \leq 1\end{array}$ \\
\hline$\alpha_{l}$ & $\begin{array}{l}\text { Rate at which the discrepancy between actual and desired } \\
\text { inventory levels is corrected, } 0 \leq \alpha_{l} \leq 1\end{array}$ \\
\hline$\alpha_{S L}$ & $\begin{array}{l}\text { The rate at which the discrepancy between actual and desired } \\
\text { supply line is corrected, } 0 \leq \alpha_{S L} \leq 1\end{array}$ \\
\hline
\end{tabular}

Assuming that the decision makers have adaptive expectations, the expected demand at time $t$ can be defined as follows:

$$
\hat{D}_{t}=\theta D_{t-1}+(1-\theta) \hat{D}_{t-1}, \quad 0 \leq \theta \leq 1 .
$$

To regulate the inventory and supply line, a negative feedback mechanism is used, that is

$$
\begin{aligned}
& A I_{t}=\alpha_{I}\left(I_{t}^{*}-I_{t}\right), \\
& A S L_{t}=\alpha_{S L}\left(S L_{t}^{*}-S L_{t}\right) .
\end{aligned}
$$

Usually $0 \leq \alpha_{S L} \leq \alpha_{I}$, and $0 \leq \beta \leq 1$ [12].

There are two approaches to determination of the desired inventory level $I_{t}^{*}$ and the desired supply line $S L_{t}^{*}$. The first one is that $I_{t}^{*}$ and $S L_{t}^{*}$ are assumed to be proportional to the expected demand and lead time [7, 10], i.e.,

$$
\begin{aligned}
& I_{t}^{*}=\delta \hat{D}_{t}, \\
& S L_{t}^{*}=\gamma \hat{D}_{t} .
\end{aligned}
$$

Hence, the decision rule of each supply chain node for the order quantity at time $t$ is defined as below

$$
O_{t}=\max \left(0, \hat{D}_{t}+A I_{t}+A S L_{t}\right)
$$

where $A I_{t}$ and $A S L_{t}$ are calculated using Eqs. (2) and (3), respectively.

The second one is to combine $I_{t}^{*}$ and $S L_{t}^{*}$ into a new variable $Q$, i.e., $Q=I_{t}^{*}+\beta S L_{t}^{*}, \beta=\alpha_{S L} / \alpha_{I}$, and set $Q$ as a constant $[5,6,8,12]$. Therefore, the decision rule for each supply chain node for the order quantity at time $t$ is defined as below $[5,6,8]$ :

$$
O_{t}=\max \left(0, \hat{D}_{t}+\alpha_{I}\left(Q-I_{t}+B_{t}-\beta S L_{t}\right)\right) .
$$

\subsection{Dynamics Model of the Supply Chain System}

Based on the above structure model, the operating mechanism and ordering policy of the supply chain system, the dynamics model is built by synthesizing several kinds of mathematics model for the beer game [5-8, 10-12, 25].

Figure 1 can be expressed by node state variables as the structure model of the supply chain system shown in Figure 2. To facilitate the model description, the following notations are introduced in Table 2.

Every variables are prescribed by a letter indicating the respective sector, i.e., using the first letter of the state variable denoting the node or sector in the supply chain, $C$ stands for customer, $R$ for retailer, $D$ for distributor, $M, W$, $P$ for sale department, production department and purchasing department of manufacturer, respectively, and $S$ for supplier.

The dynamics behaviours of the supply chain system shown in Figure 2 can be described by a set of high-dimensional piecewise-linear and nonlinear difference equations.

Only variable expressions of each sector in the manufacturer are listed as Eqs. (8)-(31), other variable expressions may be referred to Refs. $[8,23]$.

1. For the sale department of the manufacturer

The actual inventory of products

$M I_{t}=\max \left\{0, M I_{t-1}+M I S_{t-1}-M B_{t-1}-M I O_{t-1}\right\}$.

The order backlog of products

$M B_{t}=\max \left\{0, M B_{t-1}+M I O_{t-1}-M I_{t-1}-M I S_{t-1}\right\}$.

The incoming shipments of products (i.e., the production of products)

$$
\begin{aligned}
M S_{t} & =W O S_{t}=W P D_{2, t}, \\
W P D_{2, t} & =W P D_{1, t-1}, \\
W P D_{1, t} & =W P R_{t-1} .
\end{aligned}
$$

The outgoing shipments of products

$M O S_{t}=\min \left\{M I_{t-1}+M I S_{t-1}, M B_{t-1}+M I O_{t-1}\right\}$.

The incoming orders of products

$M I O_{t}=D O P_{t-1}$.

The expected demand of products

$M E D_{t}=\theta \cdot M I O_{t-1}+(1-\theta) M E D_{t-1}$.

The actual supply line of products

$M S L_{t}=W P D_{1, t}+W P D_{2, t}$. 


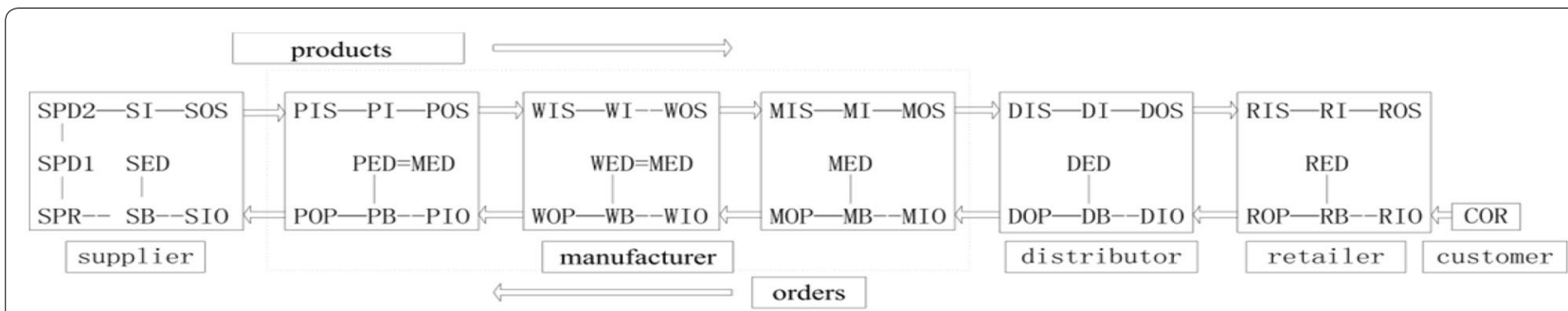

Figure 2 Structural model of the supply chain system denoted by state variables at nodes

Table 2 Notations

\begin{tabular}{ll}
\hline Symbol & Description \\
\hline I & The actual inventory level of goods \\
B & Backlog of orders \\
IS & Incoming shipments \\
OS & Outgoing shipments \\
SL & The actual supply line \\
IO & Incoming orders \\
OP & Orders placed \\
ED & The expected demand \\
PR & Production rate \\
PD1, PD2 & Production delay of one period and two periods \\
COR & The exogenous customer order rate
\end{tabular}

The orders of products placed

$$
\begin{aligned}
M O P_{t}= & \max \left\{0, M E D_{t}+\alpha_{I}\left(\delta M E D_{t}-M I_{t}\right)\right. \\
& \left.+\alpha_{S L}\left(\gamma M E D_{t}-M S L_{t}\right)\right\},
\end{aligned}
$$$$
M O P_{t}=\max \left\{0, M E D_{t}+\alpha_{I}\left(Q-M I_{t}+M B_{t}-\beta M S L_{t}\right)\right\} .
$$

2. For the production department of the manufacturer The actual inventory of work in process (WIP, i.e., unfinished products)

$W I_{t}=M S L_{t}$.

The order backlog of WIP

$W B_{t}=\max \left\{0, W B_{t-1}+W I O_{t-1}-W I_{t-1}-W I S_{t-1}\right\}$.

The volume of WIP put into production (outgoing shipments of raw material and parts)

$W I S_{t}=P O S_{t}=W P R_{t}$.

The quantity of finished WIP (i.e., the production of products)

$W O S_{t}=\min \left\{W I_{t-1}+W I S_{t-1}, W B_{t-1}+W I O_{t-1}\right\}$.

The incoming orders of WIP (i.e., the orders of products)
$W I O_{t}=M O P_{t}$.

The expected demand of WIP (i.e., the expected demand of products)

$W E D_{t}=M E D_{t}$

The actual supply line of WIP

$W S L_{t}=W I S_{t}+P I O_{t}+P B_{t}+P O S_{t}$.

The orders of WIP placed (i.e., the orders of products)

$W O P_{t}=M O P_{t}$.

3. For the purchasing department of manufacturer

The actual inventory of raw material and parts

$P I_{t}=\max \left\{0, P I_{t-1}+P I S_{t-1}-P B_{t-1}-P I O_{t-1}\right\}$.

The order backlog of raw material and parts

$P B_{t}=\max \left\{0, P B_{t-1}+P I O_{t-1}-P I_{t-1}-P I S_{t-1}\right\}$.

The incoming shipments of raw material and parts

$P I S_{t}=$ SOS $_{t-1}$.

The outgoing shipments of raw material and parts

$P O S_{t}=\min \left\{P I_{t-1}+P I S_{t-1}, P B_{t-1}+P I O_{t-1}\right\}$.

The incoming orders of raw material and parts (i.e., the orders of products)

$\mathrm{PIO}_{t}=\mathrm{WOP}_{t}$.

The expected demand of raw material and parts (i.e., the expected demand of products)

$P E D_{t}=M E D_{t}$.

The actual supply line of raw material and parts

$P S L_{t}=P I S_{t}+S I O_{t}+S B_{t}+S O S_{t}$.

The orders of raw material and parts placed (i.e., the orders of products)

$P O P_{t}=M O P_{t}$,

where subscript $t$ denotes the time period. 


\section{Ordering Policy and Operating Cost of Each Node}

3.1 Ordering Policy of Each Node and Their Combination Ordering behaviour is basic one of each node in supply chain. Different ordering policies and combination will influence not only operating state of each node, but also dynamics behaviour of the whole supply chain system. In this paper, the following nodes' ordering policies and their combinations are taken into consideration.

Order policy 1, i.e., all supply chain nodes use the same heuristic ordering policy as presented in Section 2.2, keep the ordering adjusting coefficients $\alpha_{I}$ and $\alpha_{S L}$ of these nodes consistent with each other, and determine the order quantity $O_{t}$ by Eq. (7), e.g., Eq. (15b).

Order policy 2 , i.e., all supply chain nodes use the same heuristic ordering policy, keep the ordering adjusting coefficients $\alpha_{I}, \alpha_{S L}$, the desired coverage time $\delta$ and $\gamma$ of these nodes consistent with each other $(\delta=3, \gamma=2)$, and determine the order quantity $O_{t}$ by Eq. (6), e.g., Eq. (15a).

Order policy 3 allows different ordering policies for different supply chain nodes, assuming that the manufacturer uses order policy 1, and the other nodes adopt an improved $(t, R, S)$ ordering policy or replenishing policy. Similar to the heuristic ordering policy, $(t, R, S)$ replenishing policy is also a sort of feedback based ordering policy, i.e., checking inventory level at each period, placing order if the stock level is lower than ordering point $R_{P}$ (in this study $R_{P}=20$ ), otherwise, no ordering. Let $S$ be the maximum stock capacity (in this study $S=500$ ), if the stock level $I_{t}<R_{P}$, the order quantity $O_{t}=S-I_{t}$. The improved $(t, R, S)$ replenishing policy considers the supply line $S L_{t}$, in which the actual order quantity $O_{t}=S-I_{t}-S L_{t}$.

Order policy 4 allows different ordering policies for different supply chain nodes, assuming that the supplier and the manufacturer use order policy 1 and order policy 2 , respectively, the other nodes adopt the improved $(t, R, S)$ replenishing policy.

Order policy 5 allows different ordering policies for different supply chain nodes, assuming that the manufacturer uses the improved $(t, R, S)$ replenishing policy, and the other nodes adopt order policy 1 .

There would be quite a few ordering policies and their combination in actual supply chains. In this paper, we focus on the effect of the above ordering policies and combination on the dynamics behaviour modes and operation cost.

\subsection{Operation Cost of Nodes}

There is a relation between the dynamics behaviour modes of order or inventory and the operating cost of a supply chain, where chaotic/hyperchaotic behaviour modes would bring intuitively high cost, since this kind of behaviour modes usually result in large oscillation amplitude [7]. Therefore, it is very meaningful to study the relation between behaviour modes and operating cost in the parameter space $\left(\alpha_{I}, \alpha_{S L}\right)$ for the ordering and inventory management decision of a supply chain.

Provided that the average cost of actual inventory and out-of-stock for each node in normal operation time length $T_{r}$ are calculated by Eqs. (32) and (33), respectively,

$$
\begin{aligned}
C_{T_{r}}^{I_{t}} & =\frac{C_{H}}{T_{r}} \sum_{t=1}^{T_{r}} I_{t}\left(\alpha_{I}, \alpha_{S L}\right), \\
C_{T_{r}}^{B_{t}} & =\frac{C_{B}}{T_{r}} \sum_{t=1}^{T_{r}} B_{t}\left(\alpha_{I}, \alpha_{S L}\right),
\end{aligned}
$$

where $C_{H}$ and $C_{B}$ are holding cost per unit inventory and out-of-stock cost per unit order backlog in each period, respectively, while the total average cost of each node during $T_{r}$ is

$$
C_{i}\left(\alpha_{I}, \alpha_{S L}\right)=C_{i T_{r}}^{I_{t}}+C_{i T_{r}}^{B_{t}}
$$

\section{Simulation Analysis for the Supply Chain Benchmark Model}

\subsection{Benchmark Model}

To reveal the effect of different ordering policies and combination on the dynamics behaviour and operation cost of the supply chain system model shown as Figure 2, we consider the model with the following reference scenario [5-8, 10-12] as benchmark model:

i. The customer demand mode is a step-function pattern, i.e., the demand is four units per period in the first four periods, and is increased to eight units per period from the fifth period till the end of the simulation;

ii. Ordering policy 1 is used;

iii. Order and shipments in each node equal to 4 units per week. All inventories are initialized at 12 units and all initial order backlog are zero. Moreover, for each node, $\theta=0.25, Q=17$ units of goods, $C_{H}=0.5$ and $C_{B}=1.0$ units of money.

\subsection{Simulation Design}

Provided that the simulation run length $T$ is 2000 time periods, $T_{r}=500$, ordering parameters $\left(\alpha_{I}, \alpha_{S L}\right)$ for each node are the same and constant. In the range of $0 \leq \alpha_{I} \leq 1$ and $0 \leq \alpha_{S L} \leq 1$ (increment 0.02), the actual 
inventory $I_{i, t}$ and the order backlog $B_{i, t}$, as well as the corresponding stock holding cost $C_{H i} I_{i, t}$ and out-of-stock cost $C_{H i} B_{i, t}$ are obtained by simulation, thus the effective inventory of each node $E I_{i, t}$ is calculated, where $E I_{i, t}$ is defined as the difference value of $I_{i, t}$ and $B_{i, t}$, i.e., $E I_{i, t}=I_{i, t}-B_{i, t}[4-8]$. According to the largest Lyapunov exponent LLE corresponding to $E I_{i, t}$, we can depict quantitatively the nonlinear behaviour modes of the supply chain system $[7,10,11]$, i.e., if the LLE is positive, the system is chaotic; otherwise the system is stable, periodic or quasi-periodic, where the LLE is calculated by a method of small data sets [29].

\subsection{Distribution of Behaviour Modes and Operating Cost at Each Node in the Benchmark Model}

According to Sections 2 and 3, as well as Sections 4.1 and 4.2 , we can obtain the LLE distribution $\lambda_{i \max }\left(\alpha_{I}, \alpha_{S L}\right)$ and the corresponding operating cost distribution $C_{i}\left(\alpha_{I}, \alpha_{S L}\right)$ ( $i=R, D, M, S$ ) of each node in the benchmark model, shown as Figures 3 and 4, where the values of the right color scale marked at the figure denote the LLE and the total average cost, respectively.
It can be seen from Figure 3 that,

i. From the downstream node (retailer) successively to the upstream node (supplier), the blue area $\left(\lambda_{i \max } \leq 0\right.$, corresponding to stable or periodic behaviour mode) is gradually reduced, while the non blue area $\left(\lambda_{i \max }>0\right.$, corresponding to chaotic/hyperchaotic behaviour mode) is inchmeal increased, and the color of the area is in a gradual transition from mainly green (smaller LLE value means weaker chaotic degree) to dominated yellow with saffron yellow (bigger LLE value means stronger chaotic degree), which indicates that along the supply chain from the downstream node to the upper-stream node, more and more complex the behaviours of the system appear, more and more prominent the chaotic range and degree become, i.e., there exists so-called "chaos amplification" phenomenon [10, 11];

ii. There exists so-called "chaotic corner" $[7,10]$ in the distributions of behaviour mode at each node, i.e., the region at the lower right corner of these figures

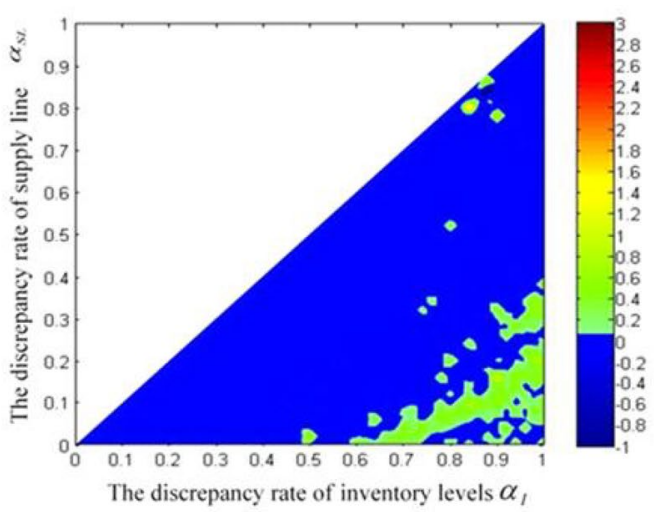

a $\lambda_{R \max }\left(\alpha_{l}, \alpha_{S L}\right)$ distribution for the retailer

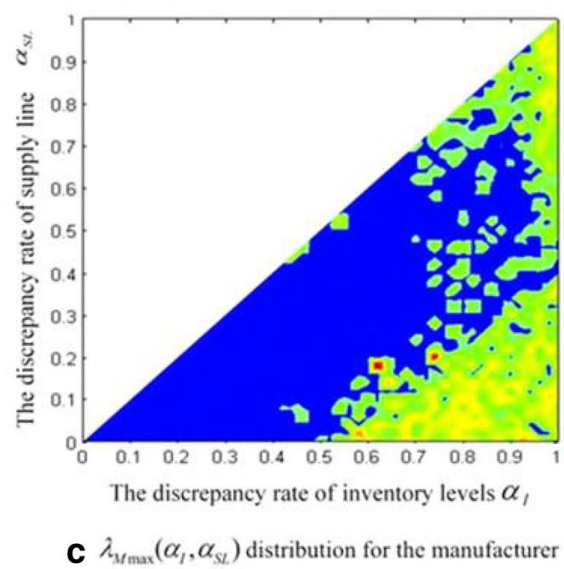

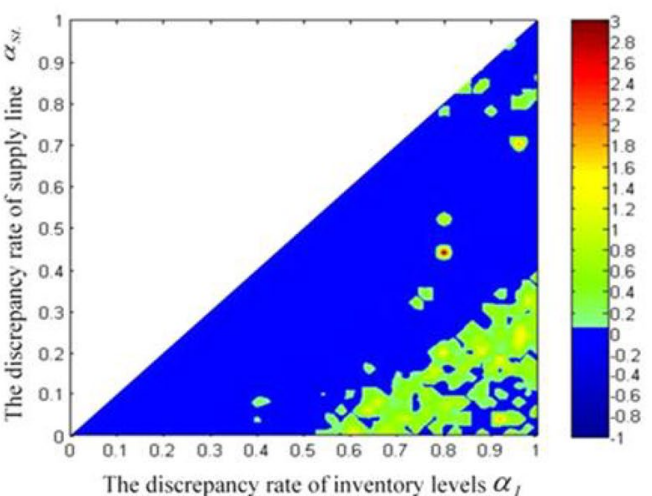

b $\lambda_{D \max }\left(\alpha_{l}, \alpha_{\mathrm{sL}}\right)$ distribution for the distributor

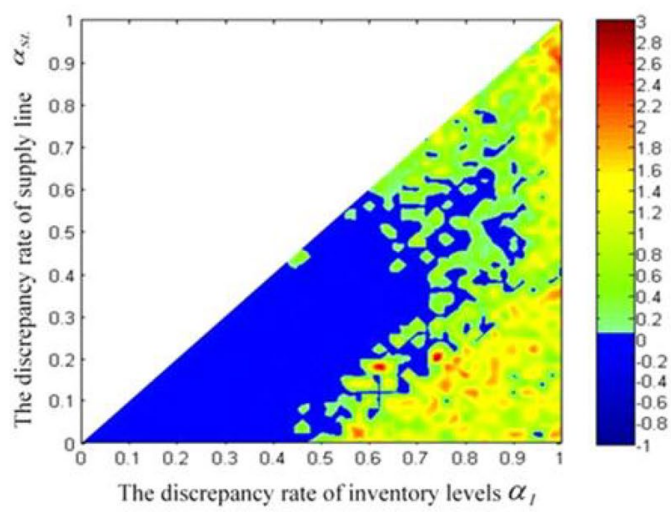

d $\lambda_{\text {Smax }}\left(\alpha_{1}, \alpha_{S L}\right)$ distribution for the supplier

Figure $3 \lambda_{i \max }$ distribution of effective inventory for each node in the parameter space $\left(\alpha_{l}, \alpha_{S L}\right)$ 


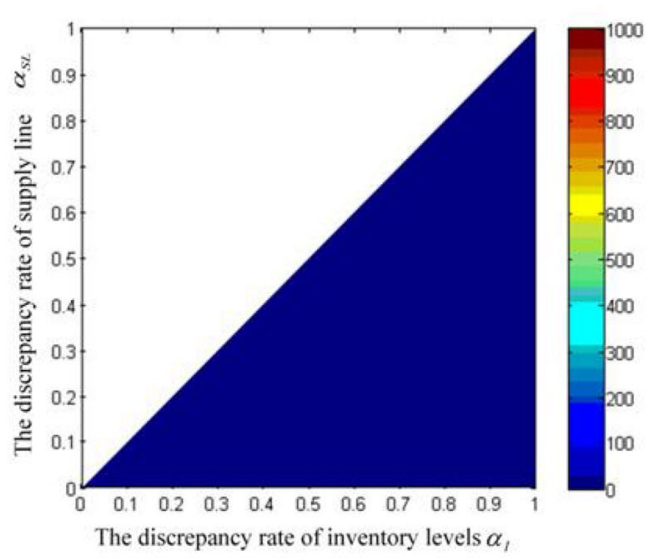

a $C_{R}\left(\alpha_{l}, \alpha_{S L}\right)$ distribution for the retailer

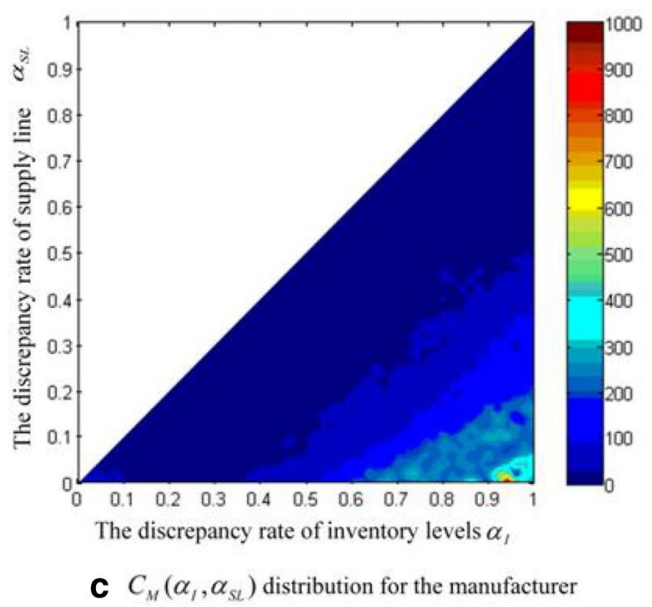

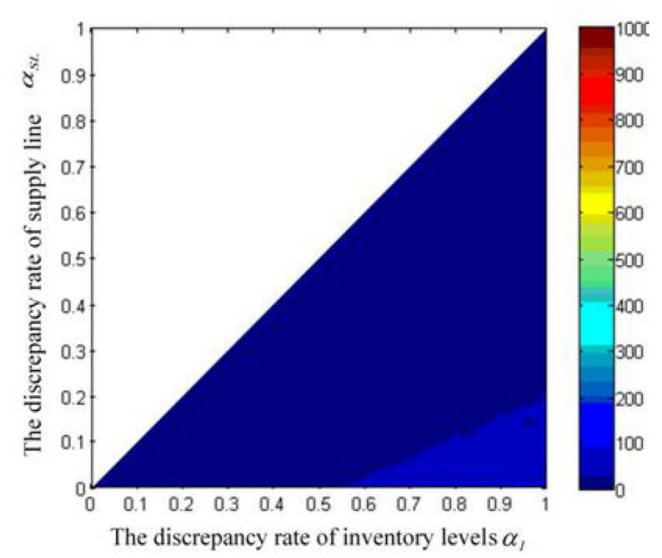

b $C_{D}\left(\alpha_{I}, \alpha_{S L}\right)$ distribution for the distributor

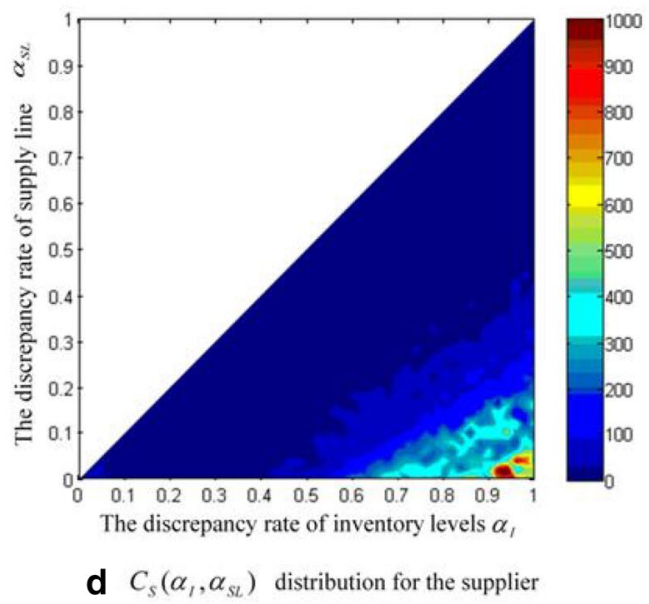

Figure 4 Total cost per period $C_{i}\left(\alpha_{l}, \alpha_{S L}\right)$ distribution for each node in the parameter space $\left(\alpha_{l,} \alpha_{S L}\right)$

(hereinafter referred to as "lower chaotic corner"), where the solutions with different properties are complicatedly mixed, and from the downstream node to the upperstream node, the "chaotic corner" region gradually diffuses into the upper right corner of these figures, which means that there exists so-called "upper chaotic corner", and is not mentioned in Refs. [7, 10, 11];

iii. Although each node belongs to a part of the supply chain system, and there exists a relation of information and goods flow between the adjacent nodes, the states and behaviours at each node seem to have relative independence, which means that for the same $\left(\alpha_{I}, \alpha_{S L}\right)$ values, not only is the magnitude of the LLE values of each node different, but also there may exist difference between the properties of the corresponding behaviour modes, i.e., it is stable for some node, but periodic or chaotic for other nodes, which can be proved by the effective inventory time series of each node and the research result of Hwarng et al. [10, 11].

It can be seen from Figure 4 that,

i. Corresponding to the "lower chaotic corner" in the distribution $\lambda_{i \max }\left(\alpha_{I}, \alpha_{S L}\right)$, there exist high cost areas at the lower right corner of the distribution $C_{i}\left(\alpha_{I}, \alpha_{S L}\right)$ of each node (hereinafter referred to as "lower loss corner"), where the operation cost and loss risk of node is higher.

ii. The distribution $C_{i}\left(\alpha_{I}, \alpha_{S L}\right)$ in the "lower loss corner" of each node shows more obvious hierarchical structure, where along the direction of the diagonal line $\alpha_{I}=\alpha_{S L}$ in the parameter space $\left(\alpha_{I}, \alpha_{S L}\right)$, an approximately parallel banded region appears, and the corresponding cost value decreases successively from the vertex at the lower right corner in the distribution $C_{i}\left(\alpha_{I}, \alpha_{S L}\right)$ along the direction 


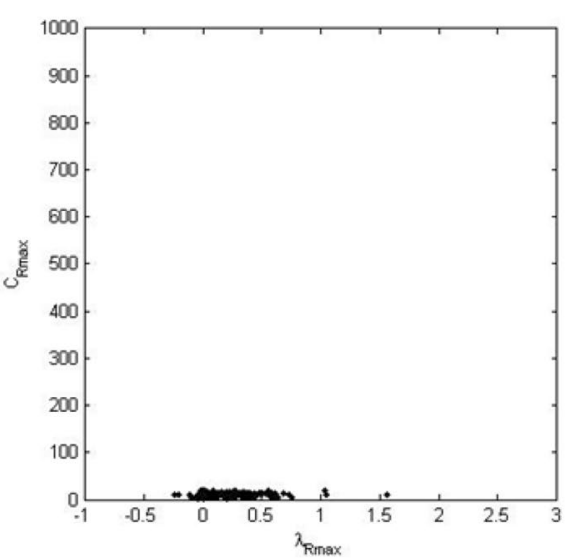

a Relation between $C_{R}$ and for the retailer $\lambda_{R \max }$

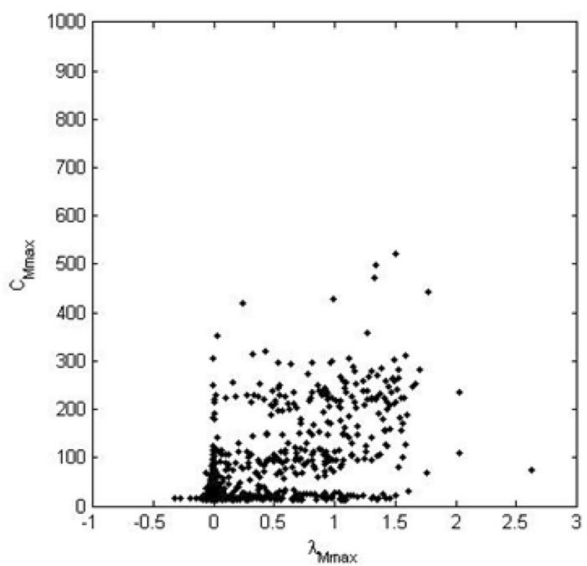

C Relation between $C_{M}$ and for the manufacturer $\lambda_{M \max }$

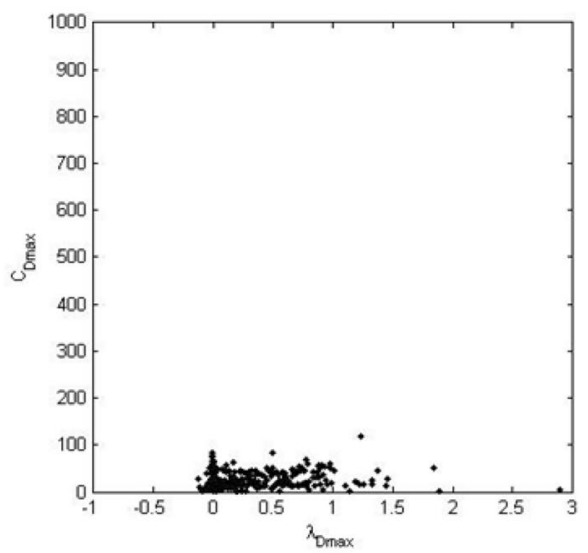

b Relation between $C_{D}$ and for the distributor $\lambda_{D \max }$

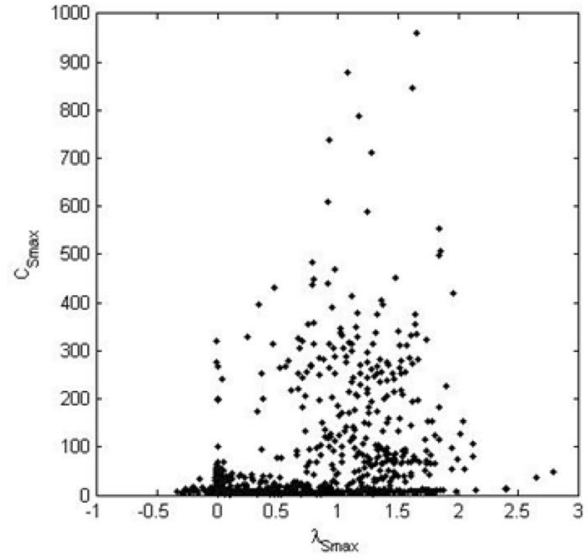

d Relation between $C_{S}$ and for the supplier $\lambda_{S \max }$

Figure 5 Relation between $C_{i}\left(\alpha_{l}, \alpha_{S L}\right)$ and $\lambda_{i \max }\left(\alpha_{l}, \alpha_{S L}\right)$ for each node

approximately perpendicular to the diagonal line $\alpha_{I}=\alpha_{S L}$.

iii. From the downstream node (retailer) successively to the upstream node (supplier), the dark blue area (lower cost region) is gradually diminishing, while the non dark blue area (higher cost region) is inchmeal expanding, or the cost value in the "lower loss corner" is gently increasing, i.e., there exists so-called bullwhip effect or "cost amplification" phenomenon, which is similar to the "chaos amplification" phenomenon in the distribution $\lambda_{\text {imax }}\left(\alpha_{I}, \alpha_{S L}\right)[10]$.

iv. In the distribution $\lambda_{i \max }\left(\alpha_{I}, \alpha_{S L}\right)$ and $C_{i}\left(\alpha_{I}, \alpha_{S L}\right)$ of each node, there exist all the corresponding "lower chaotic corner" and "lower loss corner", respectively, while only for the upstream manufacturer and supplier, there exists the "upper chaotic corner" besides the "lower chaotic corner", but there does not exist the corresponding "upper loss corner" at the upper right corner in the distribution $C_{i}\left(\alpha_{I}, \alpha_{S L}\right)$, which means that chaos is unnecessarily corresponding to high cost, or rather, the "chaos amplification" does not completely respond to the bullwhip effect or "cost amplification", and it is not mentioned in Refs. [7, 10, 11].

We can see that from the relation between $C_{i}\left(\alpha_{I}, \alpha_{S L}\right)$ and $\lambda_{i \max }\left(\alpha_{I}, \alpha_{S L}\right)$ shown as Figure 5 , the cost values of the downstream nodes (retailer and distributor) are not high despite $\lambda_{i \max }>0$ in some areas, however, these of the upstream nodes (manufacturer and supplier, especially supplier) may remarkably increase for the same $\lambda_{i \max }$. 


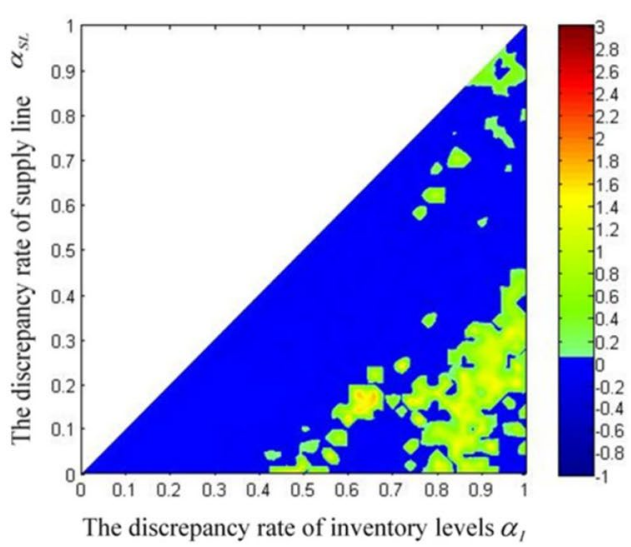

$$
\lambda_{M \max }\left(\alpha_{l}, \alpha_{S L}\right)
$$
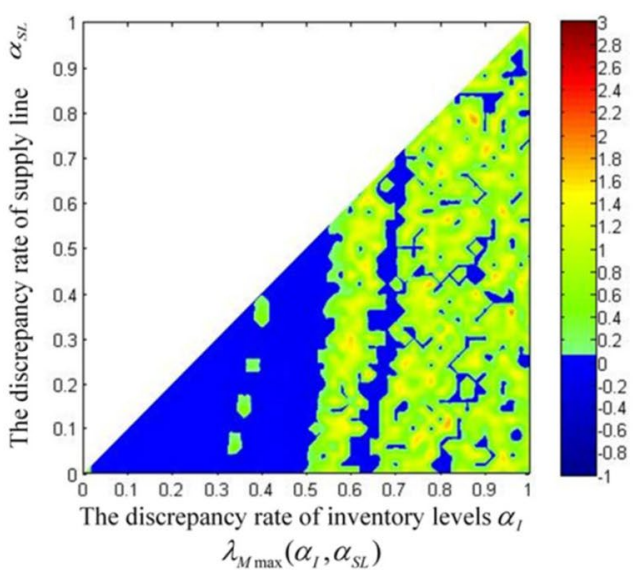

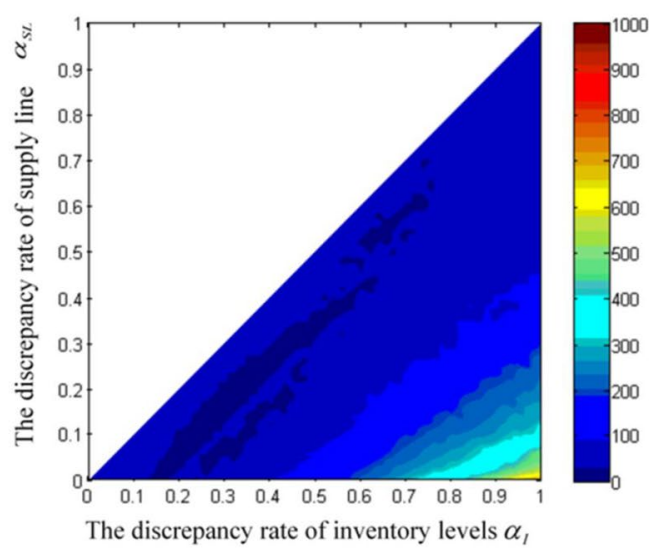

$$
C_{M}\left(\alpha_{I}, \alpha_{S L}\right)
$$

a For ordering policy 2

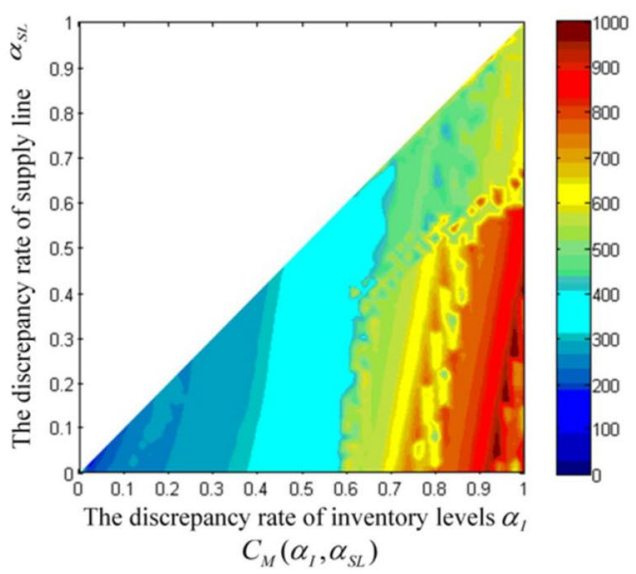

b For ordering policy 3

Figure 6 Distribution of $\lambda_{M \max }$ and $C_{M}$ for the manufacturer in the case of different ordering policies

\section{Effect of Ordering Policies on the Distribution of Behaviour Mode and Operation Cost}

Since the manufacturer is a core enterprise of the supply chain, and its cost $C_{M}\left(\alpha_{I}, \alpha_{S L}\right)$ is main part of the supply chain cost, thereinafter we take the manufacturer as a typical object of investigation to mainly study the effect of different ordering policies on its distribution $\lambda_{M \max }\left(\alpha_{I}, \alpha_{S L}\right)$ and $C_{M}\left(\alpha_{I}, \alpha_{S L}\right)$, especially on the "chaotic corner" and "loss corner", as shown in Figures 6, 7.

We can see that from the comparison between Figures 6, 7 and Figure 3(c), as well as Figure 4(c), the ordering policies have significant effect on the distribution $\lambda_{M \max }\left(\alpha_{I}, \alpha_{S L}\right)$ and $C_{M}\left(\alpha_{I}, \alpha_{S L}\right)$.

i. Not only does ordering policy 2 make the non chaotic connected region embedded in the "lower chaotic corner" obviously increasing, but also the "upper chaotic corner" and the dark blue lowest cost area in the banded region approximately parallel to $\alpha_{I}=\alpha_{S L}$ almost disappear;

ii. Ordering policy 3 causes the distribution $\lambda_{M \max }\left(\alpha_{I}, \alpha_{S L}\right)$ and $C_{M}\left(\alpha_{I}, \alpha_{S L}\right)$ to fundamentally change, in the distribution $\lambda_{M \max }\left(\alpha_{I}, \alpha_{S L}\right)$, the "lower chaotic corner" connects with the "upper chaotic corner" into a piece of region approximately parallel to $\alpha_{S L}$ axis, while the change of topological structure similar with the distribution $\lambda_{M \max }\left(\alpha_{I}, \alpha_{S L}\right)$ also occurs in the corresponding distribution $C_{M}\left(\alpha_{I}, \alpha_{S L}\right)$, where the cost values in the entire region become distinctly bigger, and the degressive direction of cost turns from the direction approximately vertical to the diagonal line $\alpha_{I}=\alpha_{S L}$ into $\alpha_{S L}$ axis;

iii. Not only does ordering policy 4 make the "lower chaotic corner" clearly minish, but also the "upper chaotic corner" vanishes, it is different from other cases that the "loss corner" usually with the larg- 

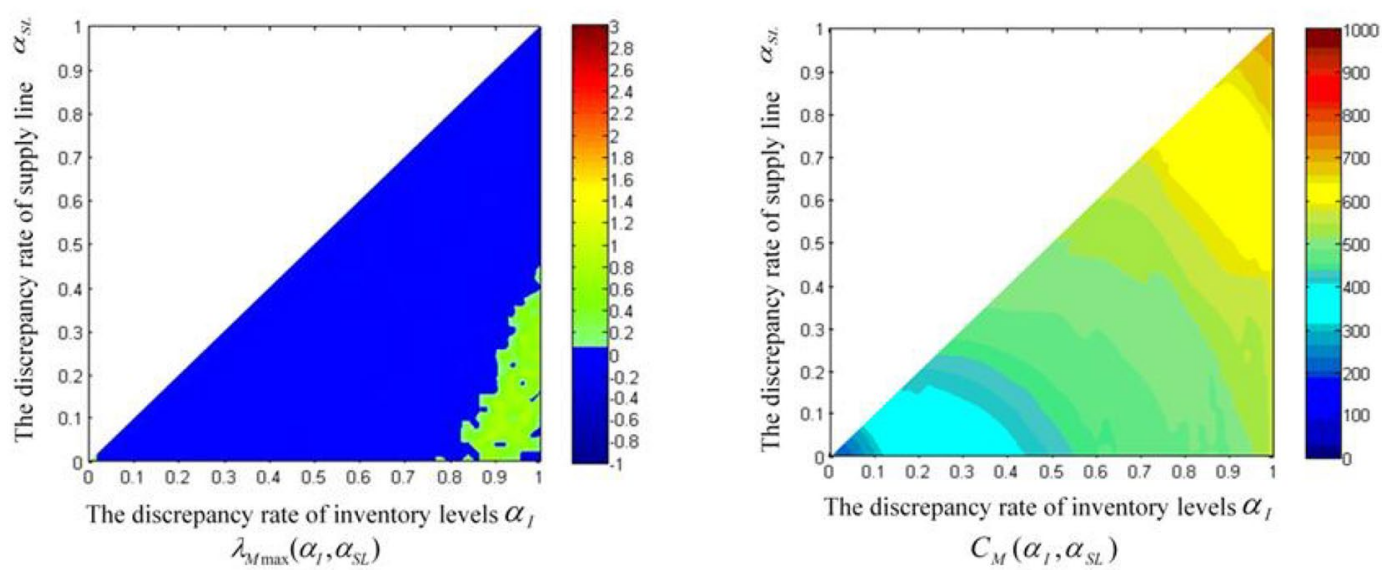

a For ordering policy 4
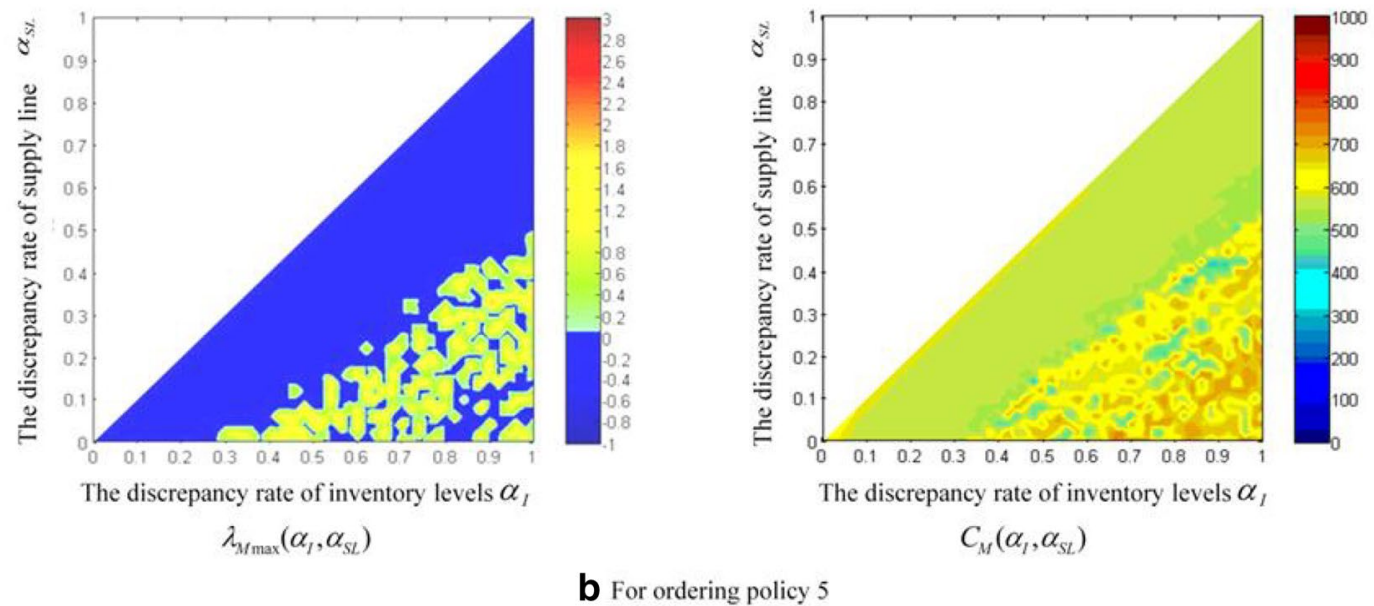

Figure 7 Distribution of $\lambda_{M \max }$ and $C_{M}$ for the manufacturer in the case of different ordering policies

est cost does not appear at the lower right corner of the distribution $C_{M}\left(\alpha_{I}, \alpha_{S L}\right)$, but transfers to the upper right corner corresponding to the "upper chaotic corner", which results in the corresponding relation between the "chaotic corner" and the "loss corner" into disappearance;

iv. Not only does ordering policy 5 make the area of the "lower chaotic corner" extend slightly, but also the "upper chaotic corner" vanishes, though the distribution $C_{M}\left(\alpha_{I}, \alpha_{S L}\right)$ still has a normal topological structure, the cost values in the entire region become remarkably bigger.

Figure 8 describes the relation between $C_{M}\left(\alpha_{I}, \alpha_{S L}\right)$ and $\lambda_{M \max }\left(\alpha_{I}, \alpha_{S L}\right)$ for the manufacturer in the case of different ordering policies. It can be seen from the comparison between Figure 8 and Figure 5(c), that the ordering policies have remarkable effect on the relation between $C_{M}\left(\alpha_{I}, \alpha_{S L}\right)$ and $\lambda_{M \max }\left(\alpha_{I}, \alpha_{S L}\right)$, where the cost values all shift evidently upward except ordering policy 2 .

\section{Discussions and Conclusions}

Ordering is the most basic behaviour in the operation of a supply chain system, while ordering policy is one of the most primary factors influencing the operating states of the system. In this paper, we have emphatically studied the effect of several ordering policies on the dynamics behaviour modes and operating cost of the supply chain system in discrete-type manufacturing industry.

The study shows that when the same ordering policy (i.e., ordering policy 1 in Section 3.1) with the original beer game model is adopted, the supply chain system model (i.e., the benchmark model in Section 4.1) in this paper has the similar distribution characteristics of dynamics behaviour modes and operating cost in the ordering parameter space with the original beer game model, i.e., in the behaviour mode distribution $\lambda_{i \max }\left(\alpha_{I}, \alpha_{S L}\right)$ so that there exist the "lower chaotic corner" $[7,10]$ and the "chaos amplification" phenomenon [10], while in the operating cost distribution $C_{i}\left(\alpha_{I}, \alpha_{S L}\right)$ there exist the "lower loss corner" corresponding to the 


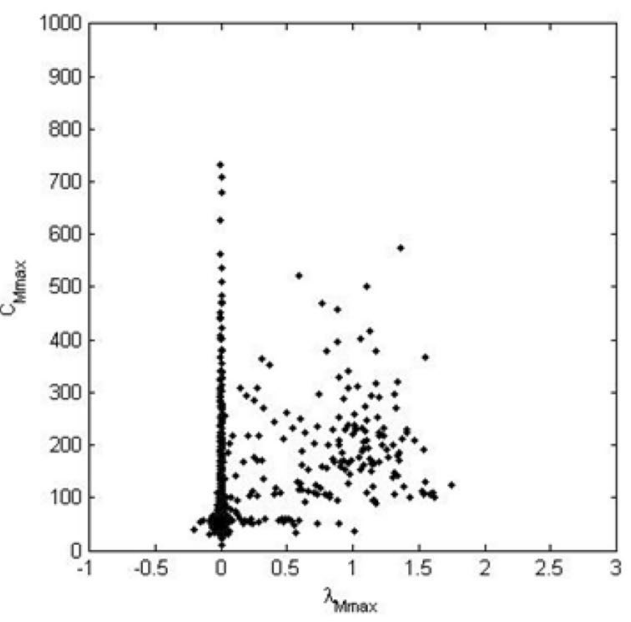

a For ordering policy 2

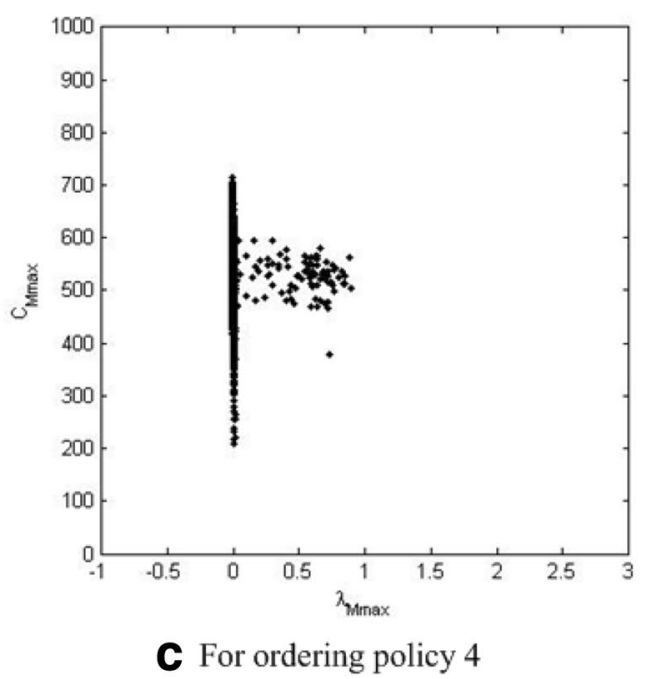

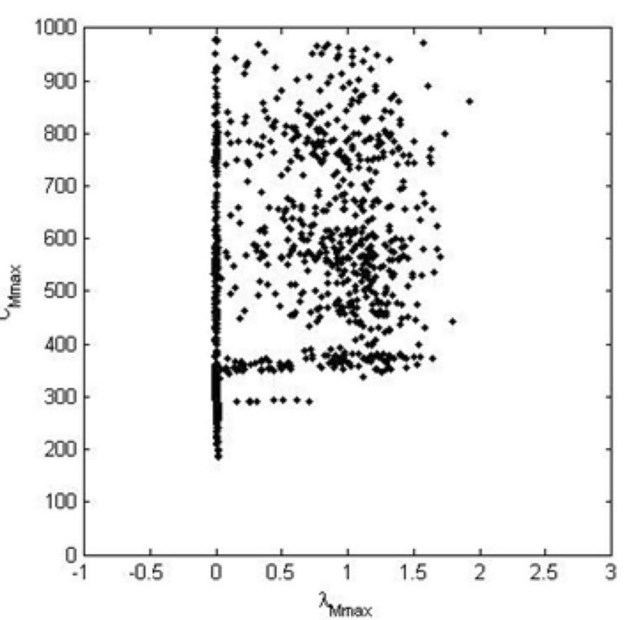

b For ordering policy 3

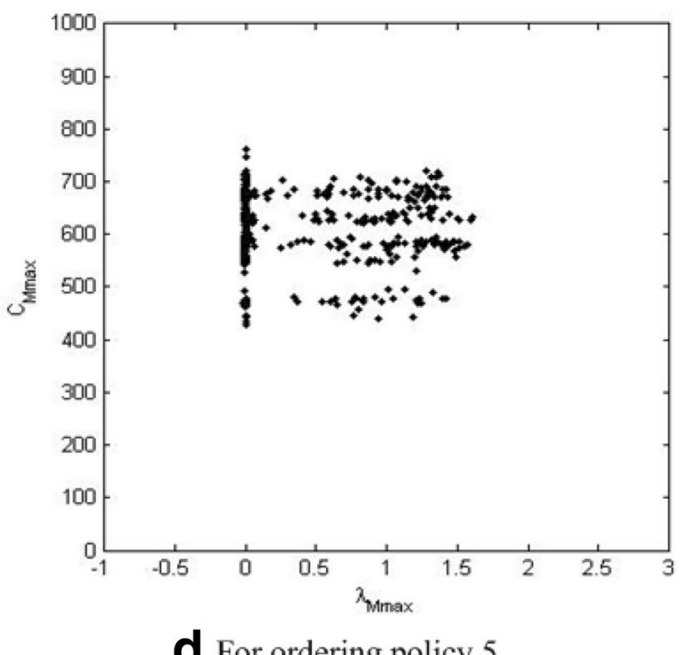

d For ordering policy 5

Figure 8 Relation between $C_{M}$ and $\lambda_{M \max }$ for the manufacturer in the case of different ordering policies

"lower chaotic corner" and the "cost amplification" phenomenon similar to the "chaos amplification". The difference, however, is that there exists still the "upper chaotic corner" in the behaviour mode distribution $\lambda_{i \max }\left(\alpha_{I}, \alpha_{S L}\right)$ of the upper stream nodes, while there does not exist the relevant "upper loss corner" in the cost distribution $C_{i}\left(\alpha_{I}, \alpha_{S L}\right)$, which means that chaos is not always corresponding to high cost, or say, the "chaos amplification" is not completely relevant to the "cost amplification".

The research also indicates that using different ordering policies in the benchmark model of the supply chain system may lead to distinct variation in the topological structure of the distribution $\lambda_{i \max }\left(\alpha_{I}, \alpha_{S L}\right)$ and $C_{i}\left(\alpha_{I}, \alpha_{S L}\right)$, which may make not only the relevant relation between the "lower chaotic corner" and the "lower loss corner" no longer exist, but also the "upper chaotic corner" disappear, and the "upper loss corner" appear, i.e., the relevant relation between the "chaotic corner" and the "loss corner" does not always exist.

In the view of reducing the cost of nodes or supply chain, we can select proper decision parameters in the policy space $\left(\alpha_{I}, \alpha_{S L}\right)$ to avoid the "chaotic corner" and the "loss corner". Since the one-to-one corresponding relation between the "chaotic corner" and the "loss corner" does not always exist, thus each node can not select the ordering parameters simply according to the relevant relation between the "chaotic corner" and the "loss corner". Adopting learning policy [24] or optimizing policy [30] is more effective method to select the ordering parameters. 


\section{Authors' Contributions}

W-PF was in charge of the whole trial; WW wrote the manuscript; WW assisted with sampling and laboratory analyses. Both authors read and approved the final manuscript.

\section{Authors' Information}

Wen Wang, born in 1966, is currently a professor at Xi'an University of Technology, China. She received her PhD degree from Xi'an University of Technology, China, in 2010. Her research interests include supply chain dynamics.

Wei-Ping Fu, born in 1957, is currently a professor and supervisor for PhD candidate at Xi'an University of Technology, China. He received his PhD degree from Xi'an Jiaotong University, China, in 1996. His research interests include nonlinear dynamics, electromechanical system dynamics and control, intelligent robot and vehicle control.

\section{Competing Interests}

The authors declare that they have no competing interests.

\section{Funding}

Supported by National Natural Science Foundation of China (Grant No.

11072192), and Shaanxi Provincial Industrial Technology Research Projects of China (Grant No. 2015GY118).

\section{Publisher's Note}

Springer Nature remains neutral with regard to jurisdictional claims in published maps and institutional affiliations.

Received: 17 September 2017 Accepted: 10 December 2018

Published online: 18 December 2018

\section{References}

[1] JW Forrester. Industrial dynamics. Cambridge, MA: The MIT Press, 1961

[2] E Mosekilde, E R Larsen. Deterministic chaos in the beer productiondistribution system. System Dynamics Review, 1988, 4(1-2): 131-147.

[3] J D Sterman. Deterministic chaos in models of human behaviour: methodological issues and experimental results. System Dynamics Review, 1988, 4(1-2): 148-178.

[4] J D Sterman, E Mosekilde, J S Thomsen. Experimental evidence of deterministic chaos in human decision making behaviour. Sloan Working Paper No. BPS-3241-91, MIT Sloan School of Management, 1991.

[5] J S Thomsen, E Mosekilde, J D Sterman. Hyperchaotic phenomena in dynamic decision making. Systems Analysis and Modelling Simulation, 1992, 9(2): 137-156

[6] O Sosnovtseva, E Mosekilde. Torus destruction and chaos-chaos intermittency in a production distribution chain. International Journal of Bifurcation and Chaos, 1997, 7: 1225-1242.

[7] E R Larsen, J D W Morecroft, J S Thomsen. Complex behaviour in a production-distribution model. European Journal of Operational Research, 1999, 119(1): 61-74.

[8] J Laugesen, E Mosekilde. Border-collision bifurcations in a dynamic management game. Computers \& Operations Research, 2006, 33(2): 464-478.

[9] E Mosekilde, J Laugesen. Nonlinear dynamic phenomena in the beer game. System Dynamics Review, 2007, 23(2-3): 229-252.

[10] H B Hwarng, N Xie. Understanding supply chain dynamics: A chaos perspective. European Journal of Operational Research, 2008, 184(3): 1163-1178.
[11] H B Hwarng, X C Yuan. Interpreting supply chain dynamics: A quasichaos perspective. European Journal of Operational Research, 2014, 233(3): 566-579.

[12] J D Sterman. Modelling managerial behaviour: Misperceptions of feedback in a dynamic decision making experiment. Management Science, 1989, 35(3): 321-339.

[13] J P Pinder. Nonlinear dynamical systems and inventory management. Managerial and Decision Economics, 1996, 17(1): 27-43.

[14] R D Wilding. The supply chain complexity triangle: uncertainty generation in the supply chain. International Journal of Physical Distribution \& Logistics Management, 1998a, 28(8): 599-616.

[15] R D Wilding. Chaos theory: Implications for supply chain management. International Journal of Logistics Management, 1998b, 9(1): 43-56.

[16] X Wang, S M Disney, J Wang. Stability analysis of constrained inventory systems with transportation delay. European Journal of Operational Research, 2012, 223(1): 86-95.

[17] Jing Wang, Xun Wang. Complex dynamic behaviours of constrained supply chain systems. Systems Engineering Theory \& Practice, 2012, 32(4): 746-751. (in Chinese).

[18] T Nowak, V Hofer. On stabilizing volatile product returns. European Journal of Operational Research, 2014, 234(3): 701-708.

[19] Y Wu, D Z Zhang. Demand fluctuation and chaotic behaviour by interaction between customers and suppliers. International Journal of Production Economics, 2007, 107(1): 250-259.

[20] J H Ma, Y Feng. The study of the chaotic behaviour in retailer's demand model. Discrete Dynamics in Nature and Society, 2008, Article ID 792031, pp. 1-12, 2008. https://doi.org/10.1155/2008/792031.

[21] Y C Wei, H W Wang, C Qi. The impact of stock-dependent demand on supply chain dynamics. Applied Mathematical Modelling, 2013, 37(18-19): 8348-8362

[22] X Wang, S M Disney, J Wang. Exploring the oscillatory dynamics of a forbidden returns inventory system. International Journal of Production Economics, 2014, 147(Part A): 3-12.

[23] Wen Wang. Study on hybrid modelling simulation and dynamics mechanism of complex supply chain systems. Xi'an: Xi'an University of Technology, 2010. (in Chinese)

[24] Wen Wang, Weiping Fu, Gengsheng Wang, et al. The complex dynamics of supply chain system with learning ability. Journal of Mechanical Engineering, 2011, 47(8): 175-182. (in Chinese)

[25] V L M Spiegler, M M Naim. Investigating sustained oscillations in nonlinear production and inventory control models. European Journal of Operational Research, 2017, 261: 572-583.

[26] W Zhang, Y M Zhang, J B Chen, et al. Research on a scheduling mechanism in a complex system based on TOC. Chaos, Solitons and Fractals, 2016, 89: 335-347.

[27] J Lin, M M Naim, L P J Gosling. The extension and exploitation of the inventory and order based production control system archetype from 1982 to 2015. International Journal of Production Economics, 2016, http:// dx.doi.org/10.1016/j.ijpe.2016.12.003.

[28] X Wang, S M Disney. The bullwhip effect: Progress, trends and directions. European Journal of Operational Research, 2016, 250: 691-701.

[29] M TRosenstein, J J Collins, C J De Luca. A practical method for calculating largest Lyapunov exponents from small data sets. Physica D: Nonlinear Phenomena, 1993, 65(1-2): 117-134.

[30] F Strozzi, J Bosch, J M Zaldivar. Beer game order policy optimization under changing customer demand. Decision Support Systems, 2007, 42(2): 2153-2163 